\title{
RECENT SURVEY AND APPLICATION OF THE SIMSUNDT SOFTWARE
}

\author{
G. Persson and H. Wirdelius \\ Department of Materials and Manufacturing Technology, \\ Chalmers University of Technology, 41296 Gothenburg, Sweden
}

\begin{abstract}
The simSUNDT software is based on a previous developed program (SUNDT). The latest version has been customized in order to generate realistic synthetic data (including a grain noise model), compatible with a number of off-line analysis software. The software consists of a Windows ${ }^{\circledR}$-based preprocessor and postprocessor together with a mathematical kernel (UTDefect), dealing with the actual mathematical modeling. The model employs various integral transforms and integral equation and enables simulations of the entire ultrasonic testing situation. The model is completely three-dimensional though the simulated component is two-dimensional, bounded by the scanning surface and a planar back surface as an option. It is of great importance that inspection methods that are applied are proper validated and that their capability of detection of cracks and defects are quantified. In order to achieve this, statistical methods such as Probability of Detection (POD) often are applied, with the ambition to estimate the detectability as a function of defect size. Despite the fact that the proposed procedure with the utilization of test pieces is very expensive, it also tends to introduce a number of possible misalignments between the actual NDT situation that is to be performed and the proposed experimental simulation. The presentation will describe the developed model that will enable simulation of a phased array NDT inspection and the ambition to use this simulation software to generate POD information. The paper also includes the most recent developments of the model including some initial experimental validation of the phased array probe model.
\end{abstract}

Keywords: Ultrasonic, Simulation, Backscattered Noise, Phased Array Model

PACS: $43.20 . \mathrm{Fn}, 43.35 . \mathrm{Zc}, 46.40 . \mathrm{Cd}$.

\section{INTRODUCTION}

Demands from mainly the Nuclear Power Industry on reliability of NDE/NDT procedures and methods have encouraged the development of simulation tools of NDT. Regulations usually demands inspection methods that have been qualified, and simulation can be used as an alternative and a complement to the experimental work that are acquired to qualify the procedures. The qualification of inspection systems includes the reliability to detect, locate, characterize and accurately determine the size of defects that may occur in the specific type of component. 
The simSUNDT is such simulation software that originally was developed in order to generate synthetic data compatible with a number of off-line analysis software [1].

\section{$\operatorname{simSUNDT}$}

The simSUNDT program is a Windows ${ }^{\circledR}-$ based preprocessor and postprocessor, see Fig. 1, together with a mathematical kernel (UTDefect, [2-6]) dealing with the actual mathematical modeling. The UTDefect computer code has been developed at the Dept. of Mechanics at Chalmers University of Technology and has been experimentally validated and verified [3-5]. The mathematical kernel employs various integral transforms and integral equation techniques to model probes and the scattering by defects, providing numerically exact solutions. The software simulates the whole testing procedure with the contact probes (of arbitrary type, angle and size) acting in pulse-echo or tandem inspection situations. The simulated test piece is at the present state restricted to be of a homogeneous and isotropic material. The model is completely three dimensional though the component is two dimensional (infinite plate with finite or infinite thickness) bounded by the scanning surface where one or two probes are scanning the object within a rectangular mesh. It is also possible to include a planar back surface, which for the strip-like crack may be tilted, but is otherwise assumed parallel to the scanning surface.

Three crack-like defects are included in the software: rectangular crack (lack of fusion), circular crack (lack of fusion) and strip-like crack (fatigue crack). Both the rectangular and the strip-like crack include the possibility to model roughness on the crack surfaces. The circular crack can also be modeled as partly closed, with the degree of closure related to the crack surface conditions (roughness) and the background pressure. The four included volumetric defects are: a spherical cavity (pore), a spherical inclusion made of an isotropic material differing from the surrounding medium (slag), a spheroid cavity (pore), and a cylindrical cavity. Either the cylindrical cavity or the circular crack can be used as reference in the simulations, i.e. modeling calibration with a side drilled hole $(\mathrm{SDH})$ or a flat bottom hole $(\mathrm{FBH})$.

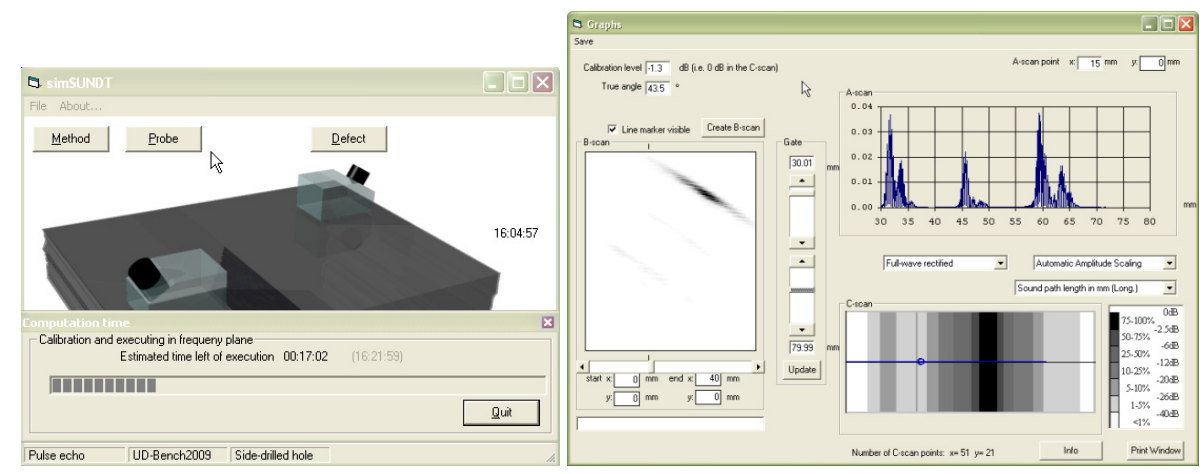

FIGURE 1. The preprocessor (left) and postprocessor (right) in the simSUNDT software. 


\section{THE GRAIN NOISE MODEL}

If realistic data are to be used in the process of generating synthetic POD there is a need for a model of the weld and the corresponding backscattered grain noise that is superposed to the defect signal that correlates better to a real inspection situation. A welding procedure does introduce different material properties in terms of anisotropy and grain size enlargement. Methods for calculating the backscattering of ultrasound due to grain boundaries have been developed during the last decade (e.g. see [7, 8]). In order to simulate this phenomenon a rather simple model is deduced. It consists of modeling grain noise by a random distribution of elastic spherical inclusions in a weld geometry where the radius of the inclusions are calculated from a one dimensional model relating welding conditions and grain size in weld heat-affected zone (HAZ). In Fig. 2 the weld geometry parameters are shown together with the Heat-Affected Zone (HAZ). No multiple scattering effects are considered in the noise signal but only a superposition of direct scattering from each defect.

In the heat-affected zone the grain sizes vary with the distance from the fusion line. If we neglect size and shape of heat source, temperature dependence of heat conductivity and specific heat, thermal loss from material surface and latent heat of fusion, the heat source can be modeled as a moving line heat source [9].

When calculating the grain size, the HAZ is divided into n parts with thickness $b_{n}$ as in Fig. 2. The fusion line is approximated by the boundary of the weld.

In order to reduce computational time it is necessary to limit the extension of the grain noise model. Grains outside the heat effect zone are thus not included. The number of inclusions must therefore be limited to avoid a non-physical discontinuity in the object (grains/no grains). On the other hand the number of defects used should ensure that the central limit theorem is satisfied. To validate this, a control volume was investigated in order to evaluate a sufficient (volume fraction) number of defects, called the level of saturation dispersion. The inclusions have the same density as the surrounding material but were provided with slightly deviating wave speeds, corresponding to $20 \%$ increase in stiffness.

Figure 3 shows a signal response that is almost linear with the number of defects for the case where the boundary of the volume is included. For the case where only the defects within the volume are included, that is where the signal from boundaries is excluded, we evidently get saturation in the signal response.

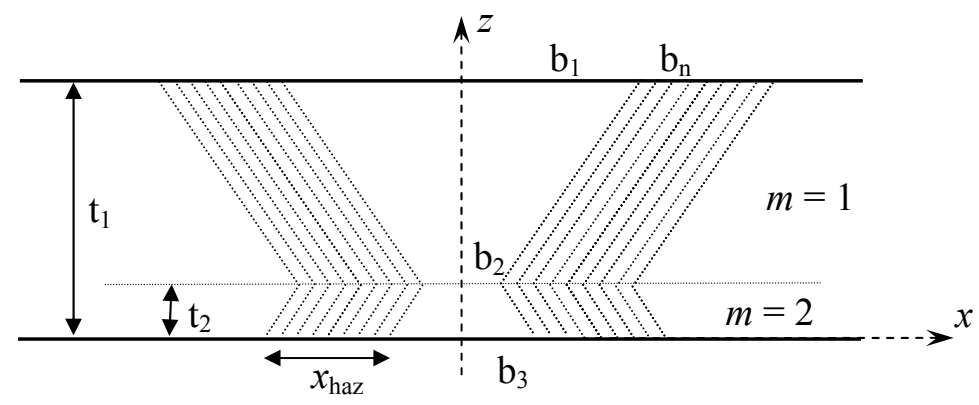

FIGURE 2. The weld geometry and HAZ. 


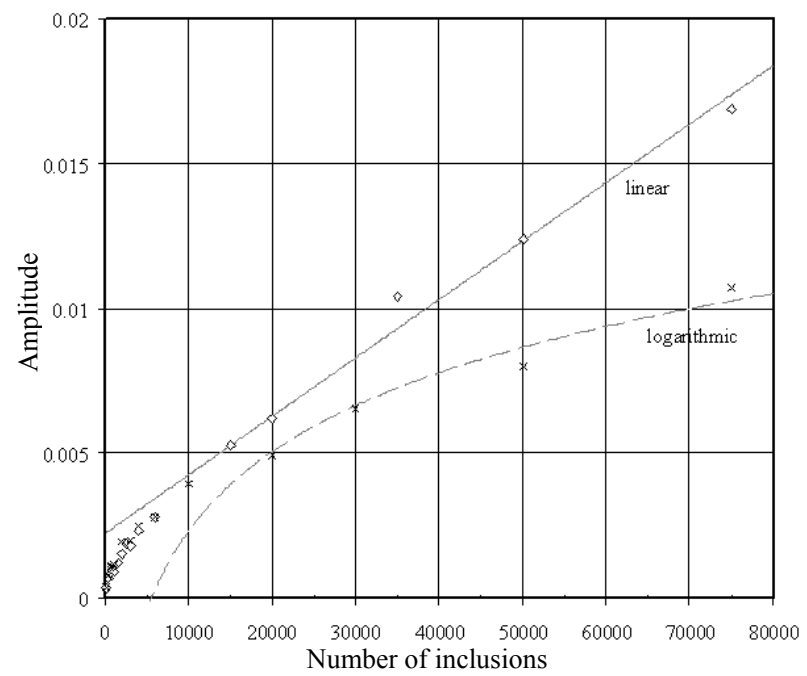

FIGURE 3. Grain noise amplitude as function of number of inclusions (the $\diamond$ marked values are rms-values based on signals from the whole volume while marked values are from within the volume).

Figure 4 shows the distribution of grain size in the weld and HAZ as a function of the $\mathrm{x}$-coordinate. Each dot represents a spherical inclusion at a specific $\mathrm{x}$-coordinate from the veldcenter, and with a particular radius. According to the evaluated number of inclusions per volume they could be distributed without taking into account to their individual size i.e. constant number per volume as shown in the left figure. However, in the computations we have chosen to let the inclusions have a constant fraction of the modeled volume in each sub region [10] as shown in the right figure.

The number of inclusions in the outer sub region is therefore defined by the level of saturation of dispersion without producing the unwanted phenomena we previously discussed. The number of inclusions in the other sub regions is provided by a constant fraction in each sub volumes.

Figure 5 shows the grain positions projected into the xy-plane. Each dot only represents the specific position in the xy-plane, the radius of spherical inclusion is not shown. The
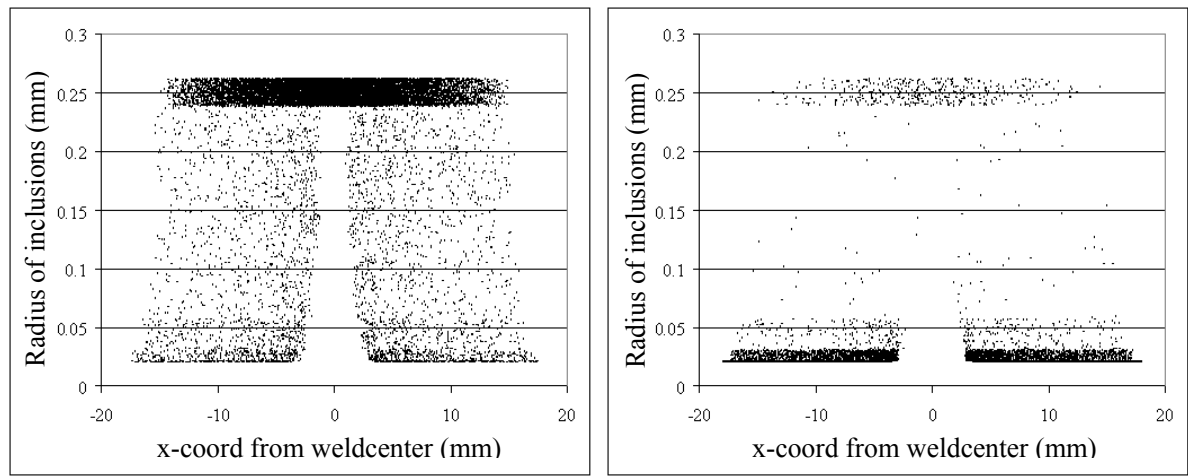

FIGURE 4. The distribution of grain size in the weld and HAZ as a function of the $\mathrm{x}$-coordinate (Fig. 2) Left with the condition of constant number per volume and right according to constant volume fraction. 

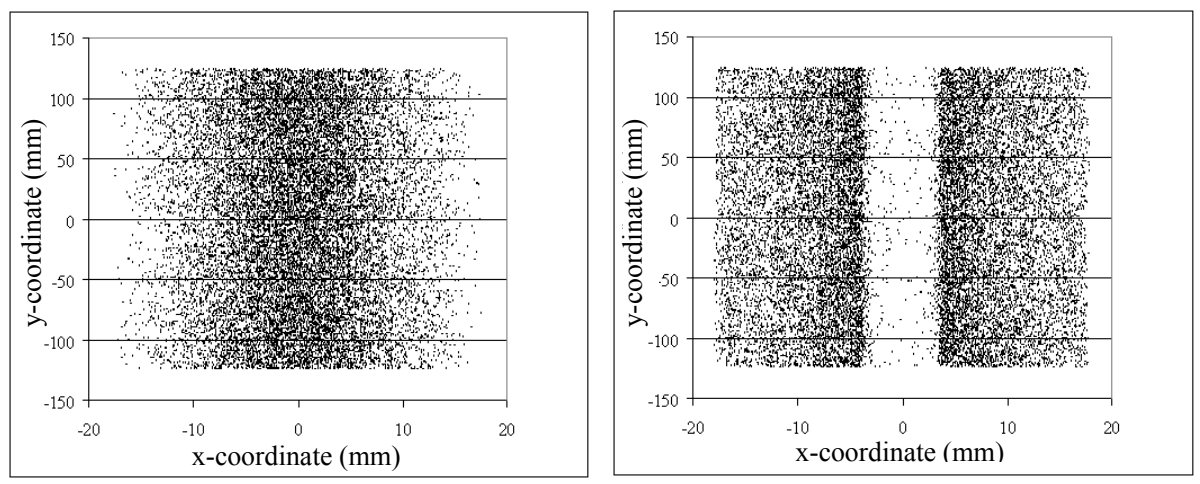

FIGURE 5. Grain position in the xy-plane. Left with the condition of constant number per volume and right according to constant volume fraction.

decrease of grain size as function of the $\mathrm{x}$-coordinate becomes obvious in the right figure since the numbers of grains that need to fulfill the condition of constant volume fraction are increased with the $\mathrm{x}$-coordinate.

\section{PHASED ARRAY PROBE}

The recent development also includes a mathematical model of a phased array probe and the implementation of it into the simSUNDT software. The phased array probe has been treated in the same way as previously was done for the conventional contact probe (see Fig. 6).

Each element is then represented by the boundary conditions that generate a plane wave, at a certain angle, in the far field. These boundary conditions (i.e. the pressure on the surface under the element) are then translated into the main coordinate system and after superposition they built up a phased array wave front (constructive phase interference) with prescribed nominal angle. Alteration of prescribed angle of each element then enables also enables a focusing effect.
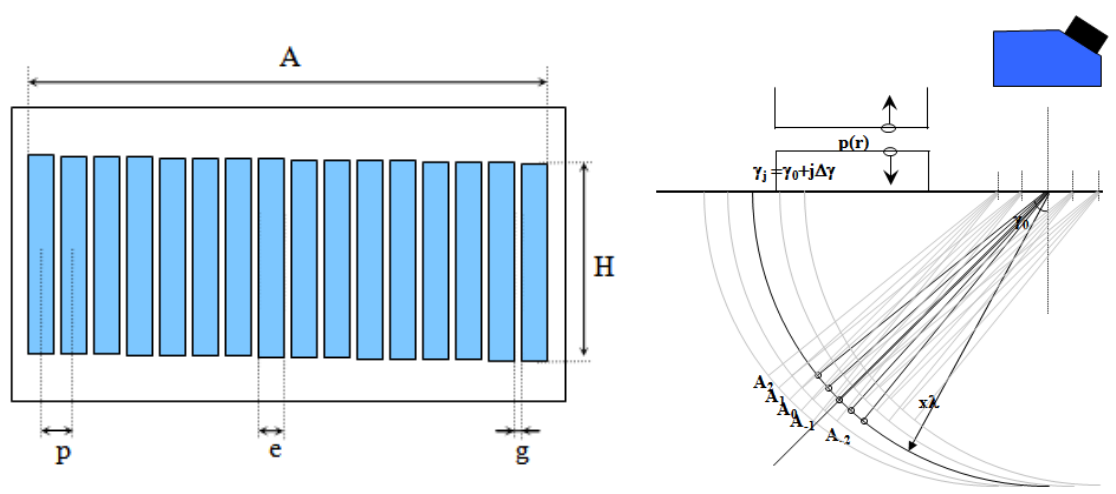

FIGURE 6. Geometry and parameters that defines the phased array probe (left), boundary conditions on the surface and far field plane wave (right). 


\section{EXPERIMENTAL RESULTS}

Within this year's benchmark an experimental study was provided that also included experimental work conducted with a well defined phased array probe on a planar block containing side-drilled holes (SDH), flat-bottom holes (FBH) and some vertical planar back wall breaking flaws (SBC) of different heights and extensions. The experimental data for these studies were conducted and generously provided by CEA in France.

Data that represents experimental signal responses, from defects that simSUNDT (version 1.2) is able to simulate, are provided in Table 1. Only 20 of the 48 elements in the linear phased array probe were activated and specification of used delay laws for these was provided as information in the documentation. The delay laws were calculated to obtain a $45^{\circ}$ longitudinal wave without any focusing effect in ferritic steel but the material in used test piece was specified as stainless steel $\left(c_{L}=5750 \mathrm{~m} / \mathrm{s}\right)$. Based upon an ad hoc assumptions of the wave speed in ferritic steel $\left(c_{L} \approx 5900 \mathrm{~m} / \mathrm{s}\right)$ and that no focusing effect would occur, $43.5^{\circ}$ was used as the nominal angle throughout most simulations.

Due to the fact that both longitudinal and transverse portions of reflected energy were provided as information it rather soon became obvious that modeled couplant conditions was less viscous than in previous validations (water instead of ultrasonic gel, see ref. [5]) which later on was confirmed by CEA. The simulation results when using 20 small elements (probes) to model the phased array probe are found in Table 2. The slots (width of $40 \mathrm{~mm}$ ) in the test piece are modeled by a surface breaking ( 2 dimensional) crack in the simulations. As can be deduced from the results the volumetric defects (SDH and $\mathrm{FBH}$ ) correlates very well to the experimental signal responses but this is not the case when it comes to the surface breaking crack. The explanation to this is probably that an infinite mathematical surface is a poor model of a more or less volumetric slot with limited dimensions though the defect model previously was verified using fatigue cracks (ref. [35]). Another explanation could be that the slots are situated at different depths than the reference defect and that the described procedure above could produce some kind of focusing effect.

TABLE 1. Experimental data (signal response in $\mathrm{dB}$ ) provided in the 2009 Benchmark. The $2 \mathrm{~mm}$ (diameter) side-drilled hole was used as reference reflector.

\begin{tabular}{|c|c|c|c|c|c|c|c|}
\hline & \multicolumn{3}{|c|}{$\mathrm{SDH}$} & FBH & \multicolumn{2}{c|}{ SBC } & \\
\cline { 2 - 7 } & $1 \mathrm{~mm}$ & $1.5 \mathrm{~mm}$ & $2 \mathrm{~mm}$ & $3 \mathrm{~mm}$ & $2 \mathrm{~mm}$ & $5 \mathrm{~mm}$ & \\
\hline $\begin{array}{c}\mathrm{L} \\
\text { direct echo }\end{array}$ & -2.6 & -1.3 & 0 (ref) & 2.8 & -10.5 & -3.6 & $\begin{array}{c}\mathrm{L} \\
\text { corner echo }\end{array}$ \\
\hline $\begin{array}{c}\mathrm{T} \\
\text { direct echo }\end{array}$ & -16.5 & -16.3 & -14.8 & & -16.2 & -7.3 & $\begin{array}{c}\mathrm{T} \\
\text { corner echo }\end{array}$ \\
\hline & & & & & -23.9 & -21.7 & $\begin{array}{c}\text { TL } \\
\text { corner echo }\end{array}$ \\
\hline
\end{tabular}

TABLE 2. Deviations $\left(20 \log \left[\mathrm{A}_{\text {simu. }} / \mathrm{A}_{\text {exp. }}\right]\right.$ ) from the experimental results (found in Table 1) when simulating the phased array with 20 elements.

\begin{tabular}{|c|c|c|c|c|c|c|c|}
\hline & \multicolumn{3}{|c|}{ SDH } & FBH & \multicolumn{2}{|c|}{ SBC } & \\
\cline { 2 - 7 } & $1 \mathrm{~mm}$ & $1.5 \mathrm{~mm}$ & $2 \mathrm{~mm}$ & $3 \mathrm{~mm}$ & $2 \mathrm{~mm}$ & $5 \mathrm{~mm}$ & \\
\hline $\begin{array}{c}\mathrm{L} \\
\text { direct echo }\end{array}$ & -0.4 & -0.1 & 0 (ref) & 1.8 & 9.6 & 9.2 & $\begin{array}{c}\mathrm{L} \\
\text { corner echo }\end{array}$ \\
\hline $\begin{array}{c}\mathrm{T} \\
\text { direct echo }\end{array}$ & -3.6 & -1.5 & -1.7 & & -2.4 & -11.3 & $\begin{array}{c}\mathrm{T} \\
\text { corner echo }\end{array}$ \\
\hline & & & & & 12.7 & 11.8 & $\begin{array}{c}\text { TL } \\
\text { corner echo }\end{array}$ \\
\hline
\end{tabular}


TABLE 3. Deviations $\left(20 \log \left[\mathrm{A}_{\text {simu }} / \mathrm{A}_{\text {exp. }}\right]\right)$ from the experimental results (found in Table 1$)$ when simulating the phased array with one single large element.

\begin{tabular}{|c|c|c|c|c|c|c|c|}
\hline & \multicolumn{3}{|c|}{ SDH } & FBH & \multicolumn{2}{|c|}{ SBC } & \\
\cline { 2 - 8 } & $1 \mathrm{~mm}$ & $1.5 \mathrm{~mm}$ & $2 \mathrm{~mm}$ & $3 \mathrm{~mm}$ & $2 \mathrm{~mm}$ & $5 \mathrm{~mm}$ & \\
\hline $\begin{array}{c}\mathrm{L} \\
\text { direct echo }\end{array}$ & -0.2 & 0.1 & 0 (ref) & 2.8 & 6.6 & 6.1 & $\begin{array}{c}\mathrm{L} \\
\text { corner echo }\end{array}$ \\
\hline $\begin{array}{c}\mathrm{T} \\
\text { direct echo }\end{array}$ & 8.9 & 11.0 & 10.5 & & 6.5 & -0.1 & $\begin{array}{c}\mathrm{T} \\
\text { corner echo }\end{array}$ \\
\hline & & & & & 1.2 & -0.5 & $\begin{array}{c}\text { TL } \\
\text { corner echo }\end{array}$ \\
\hline
\end{tabular}

TABLE 4. Deviations $\left(20 \log \left[\mathrm{A}_{\text {simu. }} / \mathrm{A}_{\text {exp. }}\right]\right)$ from the experimental results (found in Table 1$)$ when simulating the phased array with 20 elements generating a pulse with an angle of $47.5^{\circ}$.

\begin{tabular}{|c|c|c|c|c|c|c|c|}
\hline & \multicolumn{3}{|c|}{ SDH } & FBH & \multicolumn{2}{|c|}{ SBC } & \\
\cline { 2 - 7 } & $1 \mathrm{~mm}$ & $1.5 \mathrm{~mm}$ & $2 \mathrm{~mm}$ & $3 \mathrm{~mm}$ & $2 \mathrm{~mm}$ & $5 \mathrm{~mm}$ & \\
\hline $\begin{array}{c}\mathrm{L} \\
\text { direct echo }\end{array}$ & -0.3 & 0 & 0 (ref) & 2.0 & 8.2 & 9.7 & $\begin{array}{c}\mathrm{L} \\
\text { corner echo }\end{array}$ \\
\hline $\begin{array}{c}\mathrm{T} \\
\text { direct echo }\end{array}$ & -1.7 & 0.1 & 0 & & 0.2 & -9.7 & $\begin{array}{c}\mathrm{T} \\
\text { corner echo }\end{array}$ \\
\hline & & & & & 8.6 & 9.4 & $\begin{array}{c}\text { TL } \\
\text { corner echo }\end{array}$ \\
\hline
\end{tabular}

The results found in Table 3 are from simulations using only one single element which corresponds to modeling the phased array as a conventional ultrasonic contact probe. The received longitudinal contribution to the signal response still agrees fairly well while the transversal part deviates from the experimental data. This could be explained by the fact that the reflectors are situated less than one near field length from the probe. Interesting though is that the correlation between the SBC and the slot are improving by reducing the number of elements. This could also be interpreted as a consequence of some kind of focusing effect of the generated wave field. A small parametric study of the nominal angle (between 43 and 48 degree) actually resulted in a best correspondence when the angle was prescribed to be $47.5^{\circ}$ in the model (see Table 4 ).

\section{ACKNOWLEDGEMENTS}

The experimental data in this paper were conducted and generously provided by CEA in France, for this they are gratefully acknowledged.

The project is part of the TURBOKRAFT Research Programme, supported by the Swedish Energy Agency (STEM) and by Swedish industries such as Siemens Industrial Turbomachinery (SIT) and Volvo Aero Corporation (VAC).

\section{REFERENCES}

1. Wirdelius, H., SKI Report 00:29, Stockholm (2000).

2. Boström, A. and Wirdelius, H., "Ultrasonic probe modeling and nondestructive crack detection", J. Acoust. Soc. Am. 97, pp. 2836-2848 (1995).

3. Boström, A., .SKI Report 95:53, Stockholm (1995).

4. Boström, A. and Jansson, P.-Å., SKI Report 97:28, Stockholm (1997).

5. Eriksson, A.S., Boström, A. and Wirdelius, H., SKI Report 97:1, Stockholm (1997).

6. Bövik, P. and Boström, A., "A model of ultrasonic nondestructive testing for internal and subsurface cracks", J. Acoust. Soc. Am. 102, pp. 2723-2733 (1997). 
7. Rose, J. H., "Ultrasonic backscatter from microstructure", Review of Progress in Quantitative Nondestructive Evaluation. Vol. 11, Edited by D.O. Thompson and D.E. Chimenti, Plenum Press, N.Y. (1992).

8. Rose, J. H., "Theory of ultrasonic backscatter from multiphase polycrystalline solids", Review of Progress in Quantitative Nondestructive Evaluation, Vol. 12, Edited by D.O. Thompson and D.E. Chimenti, Plenum Press, N.Y. (1993).

9. Bövik, P. and Wirdelius, H., "Modelling of ultrasonic backscattering due to grain growth in a welded region", Proc. World Conf. NDT, Montreal (2004).

10. Wirdelius, Håkan: "The simSUNDT Software - Survey and Recent Development", Proc. World Congress on Ultrasonics WCU, 1, pp. 101-104 (2003). 